\title{
Significance of Follow-up in Detection of Pulmonary Metastasis of Colorectal Cancer
}

\author{
Jae Won Shin, Sun Il Lee, Hong Young Moon \\ Department of Surgery, Korea University College of Medicine, Seoul, Korea
}

Purpose: This study was performed to evaluate the effectiveness of conventional chest radiography, carcinoembrionic antigen (CEA) level and abdominal computed tomography (CT) or chest CT for early detection of pulmonary metastasis after a curative resection of colorectal cancer.

Methods: We retrospectively reviewed 84 cases of pulmonary metastasis from a group of colorectal cancer patients who had a curative surgical resection from 2000 to 2006 at the Korea University Medical Center.

Results: Stage I tumors were detected in 4 patients, stage II tumors in 18, stage III tumors in 43 and stage IV tumors in 19. The detection rates for pulmonary metastasis were $28.5 \%$ by conventional chest radiography, $40.5 \%$ by increased CEA level and $28.5 \%$ by abdominal CT or chest CT. Among them, fourteen patients underwent a radical pneumonectomy. After detection of pulmonary metastasis, the survival outcome for the patients who underwent a resection of the lung was superior to the survival outcome of the patients who did not undergo a resection of the lung ( 43.7 months vs. 17.4 months, $\mathrm{P}=$ 0.001). For patients who underwent resections of the lung, pulmonary metastasis was detected by conventional chest radiography in $2(14 \%)$ patients, by elevated CEA level in $6(42 \%)$ patients, and by abdominal CT or chest CT in $6(42 \%)$ patients.

Conclusion: Conventional chest radiography is no more useful in detecting early pulmonary metastasis after a curative colorectal surgery than a routine chest CT. Thus, we propose the use of routine chest CT for screening for lung metastasis.

\section{Keywords: Colorectal cancer; Pulmonary metastasis; Computed tomography}

\section{INTRODUCTION}

The incidence of colorectal cancer in Korea is showing a steady increase because of life-style changes. According to the statistical results from the Ministry of Health and Welfare in 2008, colorectal cancer accounts for $12 \%$ of total domestic cancer. Accordingly, interest in the prevention and treatment of colorectal cancer has increased, and numerous research results are being reported. However, approximately half of the colorectal cancer patients who are treated with a radical resection even-

Received: March 12, 2010 Accepted: August 2, 2010

Correspondence to: Hong Young Moon, M.D.

Department of Surgery, Korea University College of Medicine, Anam-dong

5-ga, Seongbuk-gu, Seoul 136-713, Korea

Tel: +82-2-2626-1140, Fax: +82-2-2626-1148

E-mail: hymoon@korea.ac.kr

(c) 2010 The Korean Society of Coloproctology

This is an open-access article distributed under the terms of the Creative Commons Attribution NonCommercial License (http://creativecommons.org/licenses/by-nc/3.0) which permits unrestricted noncommercial use, distribution, and reproduction in any medium, provided the original work is properly cited. tually die because of relapse. Thus, detecting relapse in the early stages through follow-up examination in patients who underwent a radical resection is as important as prevention or treatment of the disease.

Metastasis of colorectal cancer can happen through lymph node metastasis, hematogeneous metastasis, abdominal cavity metastasis, and implanted metastasis. Among these causes, hematogenous metastasis is known for hepatic metastasis, pulmonary metastasis, and bone metastasis. Especially, some reports insist that $10-22 \%$ of the colorectal cancer patients who undergo a radical resection get pulmonary metastasis. Among them, approximately $10 \%$ can be treated with a surgical resection $[1,2]$.

Periodic chest plain film examinations are being performed as follow-up examinations in order to discover pulmonary metastasis in colorectal cancer patients. However, because of their low sensitivity, suggestions that support the use of chest CT have been made [3]. There are different opinions on periodic follow-up examination methods considering the price and side effects of irradiation [4]. Unlike early diagnosis of re- 
lapse and increased lung excision rate increasing the survival rate, the effect of periodic follow-up examinations on the survival rate is a tenuous effect. The authors of this research retrospectively observed patients who had pulmonary metastasis after a radical resection of colorectal cancer and attempted to determine which follow-up observation methods were useful in diagnosing pulmonary metastasis. Also, follow-up observation methods to improve the patient's prognosis through early diagnosis of pulmonary metastasis, radical pulmonary resection, or systematic anticancer chemotherapy were studied.

\section{METHODS}

From January of 2000 to December of 2006, patients with colorectal cancer who were treated with a radical resection at Korea University Medical School were retrospectively surveyed based on their medical records. Among 1,124 patients 84 had pulmonary metastasis, and for them follow-up test methods were surveyed. Patients who had a radical resection while having metastasis in other organs were added to the subject group, and patients who already had pulmonary metastasis were excluded from the group.

Categorization of stage was done by using the American Joint Committee on Cancer (AJCC) cancer staging manual (6th edition). Primary colorectal cancer was categorized as cancer of the ascending colon, transverse colon, descending colon, sigmoid colon, rectosigmoid junction, or rectum based on its location. In primary cancer stage II cases where radical surgery was performed, chemotherapy based on fluorouracil was done. In patients with a stage greater than stage III, chemotherapy based on oxaliplatin and fluorouracil was used in colon cancer cases, and chemotherapy based only on fluorouracil was performed in rectal cancer cases. Radiotherapy was done along with chemotherapy in patients diagnosed with T4 rectal cancer after agreement with the patient. A chest plain film examination was executed in each hospitalization of patients going through chemotherapy. Patients with fluorouracil chemotherapy were tested after 6 treatments, and patients with oxaliplatin and fluorouracil chemotherapy were tested every 3-4 cycles. Periodic examination was done every 6 months within 1 year after surgery when the first stage was stage I. Each year, a chest plain film examination, colonoscopy, an abdominal CT, and a tumor marker test were done. After the first year, a periodic physical examination, a chest plain film examination, colonoscopy, an abdominal CT, and a tumor marker test were done for 5 years. In patients with stage greater than stage II, chemotherapy was performed, and the periodic examination and test periods were different. The primary diagnosis method for pulmonary metastasis was chest plain film examination or chest CT done because of chronic cough lasting longer than one month or haemoptysis whereas primary indicators of pulmonary metastasis were an increase in the CEA value on the peri- odic examination (increasing more than twice; CEA value doubled) in spite of no significant symptoms, a nodule observed on the chest plain film examination or on abdominal or chest CT. The final diagnosis of pulmonary metastasis included diagnosis after needle aspiration cytology or pneumonectomy and continuous increase in nodule size through numerous chest CT scans.

Pulmonary metastases were categorized as a single nodule in one lung, a multicentric nodule in one lung, or a multicentric nodule in both lungs. Patients were categorized as a radical pneumonectomy group and as a chemotherapy-due-to-impossible-pneumonectomy group, and their average survival periods were compared. Statistical analysis was done by using the SPSS ver. 15.0 (SPSS Inc., Chicago, IL, USA) program. Data were considered as statistically significant if the $\mathrm{P}$ value was less than 0.05 . The survival analysis was done by using the Kaplan-Meier method.

\section{RESULTS}

\section{Characteristics of patients}

A total of 1,124 patients underwent surgery because of colorectal cancer. One hundred seventy-seven patients were in stage I, 406 patients were in stage II, 442 patients were in stage III, and 99 patients were in stage IV. Eighty-four patients were diagnosed with pulmonary metastasis during follow-up observation (Table 1). The stages of these 84 patients during primary cancer surgery were 4 stage I patients, 18 stage II patients, 43 stage III patients, and 19 stage IV patients. Forty-two patients (50\%) were male and 42 patients (50\%) were female (Table 2 ). The average age of the patients was 60.3 years (range, 31 to 83 years). The median of follow-up observation period was 39 months (range, 3.7 to 86.9 months).

Fourteen patients underwent a radical pneumonectomy, and 70 patients were treated with chemotherapy and conservative treatment. The pneumonectomy group consisted of 10 female patients (23.8\%) and 4 male patients (9.5\%), but this difference was not statistically significant. Also, there were no statistically significance differences within the pneumonectomy as to differentiation and stage (Table 2).

Table 1. Patient characteristics

\begin{tabular}{lcccc}
\hline \multirow{2}{*}{ Stage } & $\begin{array}{c}\text { Pulmonary } \\
\text { metastases (-) }\end{array}$ & \multicolumn{2}{c}{ Pulmonary metastases } & \multirow{2}{*}{ Total } \\
\cline { 3 - 4 } & 173 & Non surgery & Surgery & \\
\hline I & 388 & 15 & 0 & $177(15.7)$ \\
II & 399 & 35 & 3 & $406(36.1)$ \\
III & 81 & 16 & 8 & $442(39.3)$ \\
IV & $1,040(92.6)$ & $84(7.4)$ & $1,124(100.0)$ & \\
Total & &
\end{tabular}

Values are presented as number or number (\%). 


\section{Pulmonary metastasis based on the location of the colorectal cancer}

All patients were treated with a radical resection for the primary cancer and adjuvant chemotherapy with 5-FU, Oxaliplatine or Irrinotecan was done in stage II or III cancer. Among 84 patients with pulmonary metastasis, 44 patients $(52.4 \%)$ had rectal cancer, 22 patients (26.2\%) had colon cancer, 13 patients (15.5\%) had right colon cancer, 3 patients $(3.6 \%)$ had left colon cancer, and 1 patient (1.2\%) had transverse colon cancer. Among the non-pneumonectomy group, 16 patients $(22.8 \%)$ had sigmoid cancer, and 38 patients $(54.4 \%)$ had rectal cancer. Among the pneumonectomy group, 6 patients (42.8\%) had sigmoid cancer, and 6 patients $(42.8 \%$ ) had rectal cancer (Table 2 ).

\section{Diagnostic method of pulmonary metastasis}

Two patients (2\%) were diagnosed with pulmonary metastasis after colorectal surgery based on abnormalities in the CEA and on the chest plain film, abdominal CT, and chest CT. Twenty-

Table 2. Characteristics of patients with pulmonary metastases

\begin{tabular}{lcc}
\hline Characteristics & Non surgery & Surgery \\
\hline Sex & & \\
Male & $38(54.3)$ & $4(28.5)$ \\
Female & $32(45.7)$ & $10(71.5)$ \\
Differential & & \\
Well & $5(7.1)$ & $3(21.4)$ \\
Moderate & $60(85.7)$ & $11(78.6)$ \\
Poorly & $3(4.3)$ & $0(0)$ \\
Mucinous & $2(2.9)$ & $0(0)$ \\
Stage & & \\
I & $4(5.7)$ & $0(0)$ \\
II & $15(21.4)$ & $3(21.4)$ \\
III & $35(50)$ & $8(57.2)$ \\
IV & $16(22.9)$ & $3(21.4)$ \\
Location & & \\
Ascending colon & $11(15.7)$ & $2(14.3)$ \\
Transverse colon & $1(1.4)$ & $0(0)$ \\
Descending colon & $3(4.3)$ & $0(0)$ \\
Sigmoid colon & $16(22.8)$ & $6(42.8)$ \\
Rectum & $38(54.4)$ & $6(42.8)$ \\
A-colon and S-colon & $1(1.4)$ & $0(0)$ \\
Total & $70(100)$ & $14(100)$ \\
\hline
\end{tabular}

Values are presented as number (\%).

Table 3. Diagnostic tools

\begin{tabular}{lc}
\hline Diagnostic tools & No. (\%) \\
\hline Symptom (+) & $2(2.0)$ \\
Symptom (-) & \\
Chest X-ray & $24(28.5)$ \\
CEA elevation & $34(40.4)$ \\
Chest computed tomography & $24(28.5)$ \\
Total & $84(100)$ \\
\hline
\end{tabular}

four patients $(28.5 \%)$ were diagnosed during periodic chest plain film test although there were no symptoms. Thirty- four patients $(40.4 \%)$ were diagnosed through abdominal or chest CT based on a continuous increase in the CEA level. Twentyfour patients $(28.5 \%)$ were diagnosed through chest CT after abnormalities were found in the abdominal CT (Table 3). Also, $88 \%$ of the patients diagnosed with pulmonary metastasis had increased CEA values.

Especially, in the pneumonectomy group, there were no cases where relapse was discovered through symptoms. Two patients (14\%) were diagnosed with relapse through chest plain film results, 6 patients $(42 \%)$ were diagnosed through increased CEA level, and 6 patients (42\%) were diagnosed through CT scanning. Thus, the surgical treatment rate was high in cases where relapse was diagnosed through CEA or CT scanning (Table 4).

\section{Pulmonary metastasis pattern comparison}

Among 84 subject patients, 7 showed a single nodule, 23 show ed a multicentric nodule in one lung, and 54 showed a multicentric nodule in both lungs. Only conservative treatment was

Table 4. Pulmonary resection according to diagnostic tools

\begin{tabular}{lc}
\hline & $\begin{array}{c}\text { Patient } \\
\text { (Surgery/Diagnosis) }\end{array}$ \\
\hline Symptom & $0 / 2(0)$ \\
Chest X-ray & $2 / 24(8.3)$ \\
CEA elevation & $6 / 34(17.6)$ \\
Chest computed tomography & $6 / 24(25.0)$ \\
\hline
\end{tabular}

Values are presented as number (\%).

Table 5. Pulmonary metastases between the surgery and the non-surgery groups

\begin{tabular}{lcccc}
\hline \multirow{2}{*}{ Single } & \multicolumn{2}{c}{ Multiple } & \multirow{2}{*}{ Total } \\
\cline { 3 - 4 } & & Unilateral & Bilateral & \\
\hline Surgery & $4(4.8)$ & $7(8.4)$ & $3(3.5)$ & $14(16.7)$ \\
Non surgery & $3(3.5)$ & $16(19.0)$ & $51(60.8)$ & $70(83.3)$ \\
Total & $7(8.3)$ & $23(27.4)$ & $54(64.3)$ & $84(100)$ \\
\hline
\end{tabular}

Values are presented as number (\%).

Table 6. Overall survival times between the surgery and the non-surgery groups after pulmonary metastases

\begin{tabular}{lccc}
\hline & \multicolumn{2}{c}{ Mean } \\
\cline { 2 - 4 } & \multirow{2}{*}{ Estimate } & \multicolumn{2}{c}{ 95\% Confidence interval } \\
\cline { 2 - 4 } & & Lower & Upper \\
\hline Non surgery & 17.4 & 13.6 & 21.2 \\
Surgery & 43.8 & 35.7 & 51.8 \\
Total (mo) & 20.9 & 16.9 & 24.9 \\
\hline
\end{tabular}




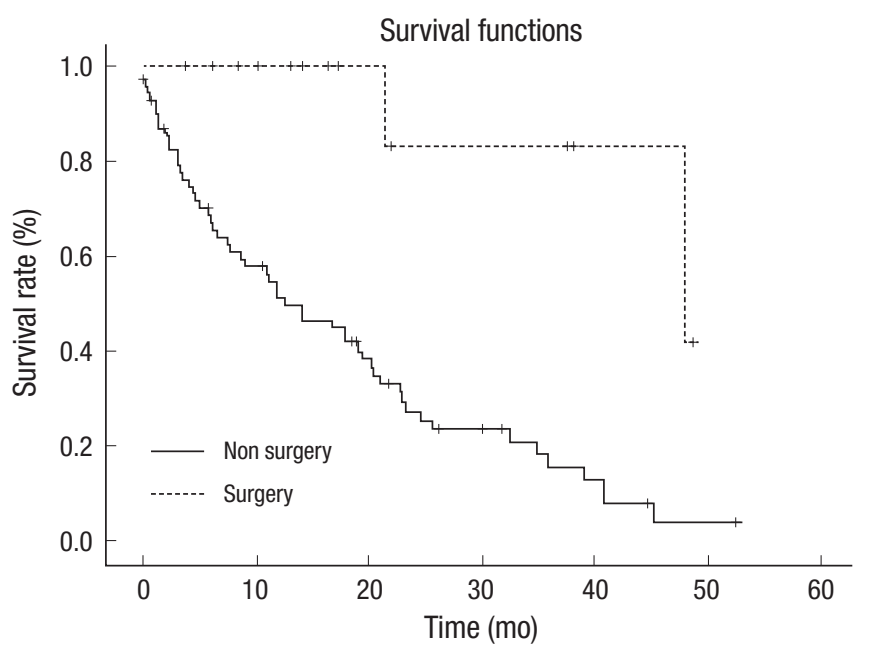

Fig. 1. Overall survival time between the surgery and the non-surgery groups after pulmonary metastases.

done in patients with a multicentric bilateral nodule. In single nodule cases, a pulmonary wedge resection was performed. A single lebectomy was performed when the nodule was limited to one lobe. Among the multicentric pulmonary metastasis group, 3 patients were in the surgical group, and 51 patients were in the non-surgical group (Table 5).

\section{Prognosis after a diagnosis of pulmonary metastasis}

Among 84 subject patients, 23 are still alive, 4 were not followed, and 57 are deceased. The average survival rate of the surgical group was 43.7 months and that of the non-surgical group was 17.4 months (Table 6, Fig. 1). This difference was statistically different (P-value, 0.001).

\section{DISCUSSION}

Among patients with primary colorectal cancer treated with surgical treatment, approximately $50 \%$ have relapse within 5 years. Among remote metastases, hepatic metastasis is the most common at $40-80 \%$. and pulmonary metastasis is the next common at approximately $10 \%[1,2]$. In this research, pulmonary metastases were observed in $7.4 \%$ of all colorectal cancer patients. Because pulmonary metastasis in colorectal cancer is often accompanied by liver, brain, bone, and many other multicentric metastases [3], a pnumonectonomy is rarely executed. However, in cases with a single pulmonary metastasis, a pneumonectomy will increase the 5-year survival rate from $14 \%$ to $78.7 \%[1,2]$. Accordingly, a single pulmonary metastasis in its early stage will result in an increased survival rate, which amplifies the importance of effective tests for early diagnosis [4].

Generally, pulmonary metastasis occurs more frequently in rectal cancer than colon cancer. This is known to be true as vein flow for the mid or the low rectum comes from system- atic vein flow. This research also showed more frequent pulmonary metastasis in rectal cancer than in colon cancer.

After a radical resection of colorectal cancer, metastasis detection is done using CEA analysis, abdomen ultrasonography, and abdominal CT. In cases of pulmonary metastasis, a chest plain film test every 6 months in the first 2-3 years is the most effective and economical method [5]. However, in recent studies, CEA analysis and chest CT have been suggested because diagnosis through the results from chest plain film often preclude surgery $[6,7]$. In this research, 2 patients (14\%) were diagnosed with pulmonary metastasis through a simple chest $\mathrm{X}$-ray, so it is thought to be difficult to diagnose metastasis in its early stages when using this technique.

CT scanning has higher rates for pulmonary metastasis diagnosis because of hematogenous metastasis, multicentric nodules, and variously sized nodules existing in both lungs in the $1 / 3$ location between the peripheral subpleural region and the lateral lung $[8,9]$. Ike et al. [10] reported that focused screening tests including CT and active pneumonectomy can increase treatment records of pulmonary metastasis in colorectal cancer Patients. Hatanaka [11] mentioned that most pulmonary metastasis patients get it within one year after surgery and that although CT scanning in all patients is inefficient, it should be done every year in patients with lung cancer and a possibility of pulmonary metastasis. On the other hand, Schoemaker et al. [12] reported that screening tests, including chest plain film and CT, do not affect the survival rate of patients with pulmonary metastasis. In pulmonary nodule diagnosis, chest CT is known to be more sensitive than a chest plain film test because the former allows $2 \mathrm{~mm}$ to $3 \mathrm{~mm}$ miniature nodules to be diagnosed. However, chest CT is also known to have a low specificity as diagnostic method of pulmonary metastasisand high cost, it exposes the patient to radiation, and it is not performed as a general screening test [13-15]. In this study, the pulmonary metastasis diagnosis rate of CT scanning was not significantly higher than that of a chest plain film test. However, bilateral pulmonary metastasis was a common metastasis pattern, and other research showed similar results. Thus, increased chest CT should enable early pulmonary metastasis diagnosis. However, no periodic chest plain film test or chest CT was done during the follow-up observation. Thus, this research did not show any difference between chest plain film test and chest CT. Surgical treatment for pulmonary metastasis is known to have a greater survival rate than non-surgical treatment $[16,17]$. Rena et al. [18] reported that surgical treatment was done based on these indications: radical surgery for primary colorectal cancer treatment, no metastasis other than pulmonary metastasis, single nodules or limited to one lung, or single nodules limited to one side of the lobe even if multicentric. Since cases where surgical treatment is possible have relatively less developed metastases, it is difficult to compare the survival rate. However, the surgical group had a much greater survival rate than the 
non-surgical group. Therefore, early diagnosis of pulmonary metastasis for surgical treatment is thought to be important.

Higashiyama et al. [19] insists that an increase in the CEA level is an important prognostic factor among surgically-treated colorectal cancer patients with pulmonary metastasis. In this research, metastasis diagnosis rate based on increased CEA level (42\%) or CT scanning (42\%) was higher than that based on chest plain film tests (14\%). This shows that, as follow-up tests, continuous CEA testing and CT scanning are necessary for colorectal cancer patients rather than chest plain film tests.

The 2009 National Comprehensive Cancer Network (NCCN) guideline also suggests CEA level testing and CT scanning as follow-up tests for postoperative colorectal cancer patients. Hence, postoperative CEA level testing and CT scanning are thought to be reasonable.

The limitations of this research are the small subject group, the lack of specific tests in past follow-up testing, and difference in lengths of the follow-up observation periods. Recently, chemotherapy has been used, along with periodic chest plain film tests or CT scans, for colorectal cancer patients with greater than stage II cancer. Therefore, continuous analysis should enable effective tests for diagnosing pulmonary metastasis of colorectal cancer.

Pulmonary metastasis after radical surgery for colorectal cancer was more frequent in patients with rectal cancer. CEA level and CT can be considered as effective follow-up tests in pulmonary metastasis diagnosis because of their having a greater associated radical pneumonectomy rate, and a radical pneumonectomy in pulmonary metastasis patients increases the long-term survival rate. Accordingly, periodic CEA level analysis and CT scanning are effective pulmonary metastasis follow-up tests for colorectal cancer patients (especially patients with rectal cancer) and should increase early diagnosis and survival.

\section{CONFLICT OF INTEREST}

No potential conflict of interest relevant to this article was reported.

\section{REFERENCES}

1. Moore KH, McCaughan BC. Surgical resection for pulmonary metastases from colorectal cancer. ANZ J Surg 2001;71:143-6.

2. Inoue M, Kotake Y, Nakagawa K, Fujiwara K, Fukuhara K, Yasumitsu T. Surgery for pulmonary metastases from colorectal carcinoma. Ann Thorac Surg 2000;70:380-3.

3. Benjamin MS, Drucker EA, McLoud TC, Shepard JA. Small pulmonary nodules: detection at chest CT and outcome. Radiology 2003;226:489-93.

4. Chang AE, Schaner EG, Conkle DM, Flye MW, Doppman JL, Rosenberg SA. Evaluation of computed tomography in the detection of pulmonary metastases: a prospective study. Cancer
1979;43:913-6.

5. Castells A, Bessa X, Daniels M, Ascaso C, Lacy AM, Garcia-Valdecasas JC, et al. Value of postoperative surveillance after radical surgery for colorectal cancer: results of a cohort study. Dis Colon Rectum 1998;41:714-23.

6. Choi HS, Youk EG, Park YJ, Park KJ, Lee JW, Kim JH, et al. Pulmonary resection for lung metastases from colorectal cancer. J Korean Soc Coloproctol 1999;15:113-9.

7. Kievit J. Colorectal cancer follow-up: a reassessment of empirical evidence on effectiveness. Eur J Surg Oncol 2000;26:322-8.

8. Willis RA. The spread of tumours in the human body. 3rd ed. London: Butterworths; 1973.

9. Spencer H. Pathology of the lung. 4th ed. Oxford: Pergamon Press; 1985.

10. Ike H, Shimada H, Ohki S, Togo S, Yamaguchi S, Ichikawa Y. Results of aggressive resection of lung metastases from colorectal carcinoma detected by intensive follow-up. Dis Colon Rectum 2002;45:468-73.

11. Hatanaka K. CT evaluation of pulmonary metastases: usefulness in comparison with chest radiography. Nippon Igaku Hoshasen Gakkai Zasshi 1999;59:663-9.

12. Schoemaker D, Black R, Giles L, Toouli J. Yearly colonoscopy, liver $\mathrm{CT}$, and chest radiography do not influence 5 -year survival of colorectal cancer patients. Gastroenterology 1998;114:7-14.

13. Muhm JR, Brown LR, Crowe JK, Sheedy PF 2nd, Hattery RR, Stephens DH. Comparison of whole lung tomography and computed tomography for detecting pulmonary nodules. AJR Am J Roentgenol 1978;131:981-4.

14. Schaner EG, Chang AE, Doppman JL, Conkle DM, Flye MW, Rosenberg SA. Comparison of computed and conventional whole lung tomography in detecting pulmonary nodules: a prospective radiologic-pathologic study. AJR Am J Roentgenol 1978;131:51-4.

15. Sobue T, Moriyama N, Kaneko M, Kusumoto M, Kobayashi T, Tsuchiya R, et al. Screening for lung cancer with low-dose helical computed tomography: anti-lung cancer association project. J Clin Oncol 2002;20:911-20.

16. Baron O, Amini M, Duveau D, Despins P, Sagan CA, Michaud JL. Surgical resection of pulmonary metastases from colorectal carcinoma: five-year survival and main prognostic factors. Eur J Cardiothorac Surg 1996;10:347-51.

17. Pihl E, Hughes ES, McDermott FT, Johnson WR, Katrivessis H. Lung recurrence after curative surgery for colorectal cancer. Dis Colon Rectum 1987;30:417-9.

18. Rena O, Casadio C, Viano F, Cristofori R, Ruffini E, Filosso PL, et al. Pulmonary resection for metastases from colorectal cancer: factors influencing prognosis. Twenty-year experience. Eur J Cardiothorac Surg 2002;21:906-12.

19. Higashiyama M, Kodama K, Higaki N, Takami K, Murata K, Kameyama $M$, et al. Surgery for pulmonary metastases from colorectal cancer: the importance of prethoracotomy serum carcinoembryonic antigen as an indicator of prognosis. Jpn J Thorac Cardiovasc Surg 2003;51:289-96. 